\title{
EDITORIAL
}

\section{SURVEILLANCE AND PREVENTION OF NON- COMMUNICABLE DISEASES (NCDs) IN BANGLADESH}

Only about 70 years back, the prime concern of physician was infectious diseases which were the leading cause of death for centuries. By the dawn of the third millennium, the entire world is drifting towards the non communicable diseases (NCDs). By 2020 , it is predicted that these diseases will be causing seven out of every 10 deaths in developing countries where, the transition imposes more constraints to deal with the double burden of infective and non-infective diseases in a poor environment characterized by illhealth systems. Many of the non communicable diseases can be prevented by tackling associated risk factors. The development and implementation of NCDs prevention polices in the developing countries like Bangladesh, is a multidimensional challenge. ${ }^{1}$

Non-communicable diseases have a considerable toll on individuals, societies and health systems. Located in South Asia, Bangladesh has a population of about 150 million and a per-capita health expenditure of US $\$ 64$ will not be sufficient enough to combat with this condition. ${ }^{2}$

The world Health Report 2002 had illustrated that NCDs accounts for almost $60 \%$ of deaths and $46 \%$ of the global burden of disease. If present trends continue, by 2020 , these diseases are expected to account for $73 \%$ of deaths and $60 \%$ of disease burden. ${ }^{3}$

NCDs are important cause of disease burden, morbidity and mortality. At least $25 \%$ of the deaths in primary and secondary government health facilities are caused by these diseases. Presently, Bangladesh does not have a community-based public health program for NCDs. Only hospital-based information, although poor, is available. The major limitations which are being faced for initiation of surveillance on NCDs include lack of advocacy, lack of logistic and other facilities as well as difficulties in the generating resources for newer initiatives. $^{4}$

Globalization, unplanned urbanization and environmental and life style factors on a background of over population have been contributing significantly to increase burden of NCDs. It is estimated that by 2010, NCDs will be responsible for 59\% of deaths, compared to $40 \%$ in 1990 . Although the country is lacking a good surveillance system, the magnitude of NCDs is considered to be fairly high in Bangladesh. In 2000, top ten causes of death in Bangladesh included asthma/COPD, stroke, heart diseases, hypertension and diabetes. ${ }^{5}$

Conventionally NCDs include cardiovascular disease, stroke, diabetes, cancer and chronic respiratory diseases. However, our national plan includes other commonly prevalent non-communicable diseases or conditions like mental illnesses, injuries and blindness because of the country's requirements to be addressed through synchronized public health measures within a common strategic framework. Surveillance, prevention and management of injuries, mental illnesses and blindness could be incorporated in to this platform for a cost effective outcome.

NCD surveillance is a new endeavor in Bangladesh. Therefore, its plan of implementation needs to be simple and easily administrable. Systematic collection, analysis and interpretation of health data and the timely dissemination of this data to policymakers and others are required to implement an effective surveillance system. WHO is pursuing surveillance as part of a global strategy for preventing and controlling NCDs. The WHO STEP wise approach to surveillance of NCD risk factors uses a standard survey instrument and a methodology that can be adapted to different country resource settings and help to build country capacity. ${ }^{6}$

The key to control of the global epidemic of NCDs is primary prevention. Prevention of NCDs through promotion of healthy life style is necessary during all phase of life. Action to reduce NCDs should focus on preventing and controlling the risk factors in an integrated manner. Intervention at all levels of society, from communities to governments, private organizations and nongovernmental groups, is essential for prevention since the risk factors are 
entrenched in the framework of society and influenced by many areas of national policy. NCDs are linked to various modifiable risk factors which are intermingled with peoples' daily lifestyle include tobacco use, alcohol abuse, unhealthy diet, physical inactivity, obesity, raised blood pressure and blood glucose, abnormal blood lipid, environmental carcinogens etc. So, our approach should comprise an effective prevention programme of NCDs by amplifying awareness of people about a perfect and healthy lifestyle.

\section{Dr. Md. Abul Faiz}

PhD, FCPS, FRCP

Professor of Medicine and

Directorate General, Health Services, Health Directorate Government of People's Republic of Bangladesh

\section{References:}

1. Abdesslam Boutayeb, Saber Boutayeb. The burden of non communicable diseases in developing countries. Int J Equity Health 2005; 4: 2.
2. World Health Organization. Core Health Indicators 2007 Database. Available at: www.who.int/whosis/ database. Accessed July,2007.

3. World Health Organization. The World Health Report 2002. Available at: www.who.int/whr/2002/ annex/en/.

4. World Health Organization Bangladesh. NCD \& Mental Health. Surveillance, Prevention and Management of Non-communicable Diseases. Available at: www.whoban.org/nc_dis_surv.html. Accessed August, 2008.

5. Strategic plan for Surveillance and prevention of non-communicable diseases in Bangladesh,2007 2010. Director General of Health Services, Ministry of Health and Family Welfare,2007.

6. World Health Organization. Noncommunicable Disease Surveillance. Available at: www.who.int/ ncd_surveillance/en/. Accessed 2008. 